\title{
Hybrid conjugate gradient parameter for solving symmetric systems of nonlinear equations
}

\author{
M. K. Dauda ${ }^{1}$, Mustafa Mamat ${ }^{2}$, Mohamad A. Mohamed ${ }^{3}$, Nor Shamsidah Amir Hamzah \\ ${ }_{1,2,3}$ Faculty of Informatics and Computing, Universiti Sultan Zainal Abidin, Terengganu, Malaysia \\ ${ }^{1}$ Department of Mathematical Sciences, Kaduna State University, Nigeria \\ ${ }^{4}$ Faculty of Applied Science and Technology, Universiti Tun Hussien Onn, Malaysia
}

\begin{tabular}{l} 
Article Info \\
\hline Article history: \\
Received Nov 1, 2018 \\
Revised Feb 6, 2019 \\
Accepted Mar 15, 2019 \\
\hline
\end{tabular}

\section{Keywords:}

Conjugate Gradient

Derivative Free

Hybrid

Nonlinear Equations

Symmetric System

\begin{abstract}
Mathematical models from recent research are mostly nonlinear equations in nature. Numerical solutions to such systems are widely needed and applied in those areas of mathematics. Although, in recent years, this field received serious attentions and new approach were discovered, but yet the efficiency of the previous versions suffers setback. This article gives a new hybrid conjugate gradient parameter, the method is derivative-free and analyzed with an effective inexact line search in a given conditions. Theoretical proofs show that the proposed method retains the sufficient descent and global convergence properties of the original CG methods. The proposed method is tested on a set of test functions, then compared to the two previous classical CG-parameter that resulted the given method, and its performance is given based on number of iterations and CPU time. The numerical results show that the new proposed method is efficient and effective amongst all the methods tested. The graphical representation of the result justify our findings. The computational result indicates that the new hybrid conjugate gradient parameter is suitable and capable for solving symmetric systems of nonlinear equations.
\end{abstract}

Copyright $(2019$ Institute of Advanced Engineering and Science. All rights reserved.

\section{Corresponding Author:}

Mustafa Mamat,

Faculty of Informatics and Computing,

Universiti Sultan Zainal Abidin, Terengganu, Malaysia.

Email: musmat567@gmail.com

\section{INTRODUCTION}

In recent years, many researchers [1-5] focused on the techniques which resulted to emergence of several CG formulas coefficient ( $\beta \_\mathrm{k}$ ) in solving unconstrained optimization problems, but hybridizing the $C G$ coefficient $\left(\beta \_\right.$k) gives so many advantages. Hybrid CG parameters are basically designed based on an adaptive switch from a CG parameter, these includes the hybridizations of PRP and FR methods in proposed by TouatiAhmed and Storey [6], Hu and Storey [7] and Gilbert and Nocedal [1], the hybridizations of HS and DY methods proposed by Dai and Yuan [8] and the hybridization of LS and CD methods proposed by TouatiAhmed and Storey [6]. In the hybrid CG methods of [6, 8], the CG parameter is computed based on discrete combinations of the CG parameters of the two categories. Recently, Andrei [9-13] proposed several efficient hybrid CG methods based on convex combinations of the CG parameters of the two categories which are continuous combinations. More exactly, in [9] a hybridization of HS and DY methods has been proposed in which the hybridization parameter is computed based on the standard secant (quasi-Newton) equation [14, 15]. In [10], using an acceleration scheme, Andrei proposed another hybridization of HS and DY methods in which the hybridization parameter is computed based on a modified secant equation proposed by Li et al. [3]. The hybridization parameter in our methods is computed from a modified secant equation obtained based on the search direction of the Hager-Zhang nonlinear CG method. The survey by Hager and Zhang (2006) discussed 
extensively on some methods with special emphasis on their global convergence. The main aim of this proposed hybrid method is to find out new formula for conjugate methods such that they are not only globally convergent for general functions but also have good numerical performance.

\section{DERIVATION OF THE HYBRID CONJUGATE GRADIENT PARAMETER FOR SOLVING SYMMETRIC SYSTEMS OF NONLINEAR EQUATIONS}

In this section, the new formula was derived from [16] and [17], the resulting hybridizations formula is given below. The attractive features of these methods are to attain good computational performance and to maintain the attractive feature of strong global convergence. In order to take advantage of these, a hybridizations of these methods is carried out using the Andrei's approach together with the nonnegative restriction of the CG parameters suggested by Powell [5].

From [16], the CG parameter is given by

$$
\beta_{k_{1}}=\frac{\left(\theta_{k} y_{k}-s_{k}\right)^{T} g_{k+1}}{y_{k}^{T} \theta_{k}}, \theta_{k}=\frac{s_{k}^{T} s_{k}}{s_{k}^{T} y_{k}} k=1,2,3, \cdots
$$

Also, from [17], the CG parameter is given by

$$
\beta_{k_{2}}=\frac{\left(y_{k}-s_{k}\right)^{T} F\left(x_{k+1}\right)}{y_{k}^{T} d_{k}}, k=1,2,3, \cdots
$$

Considering the concept of $[18,18,20]$, the new $\mathrm{CG}$ parameter $\beta_{k}$ is given by:

$\beta_{k}=(1-\alpha)+\alpha \beta_{k_{2}}$, where $\alpha$ is the hybridization parameter and is a scalar satisfying $0<\alpha<1$. If $\alpha=1$, then $\beta_{k}=\beta_{k_{2}}$ while $\beta_{k}=\beta_{k_{1}}$ if $\alpha=0$.

Thus,

$$
\beta_{k}=(1-\alpha) \frac{\left(\theta_{k} y_{k}-s_{k}\right)^{T} g_{k+1}}{y_{k}^{T} \theta_{k}}+\alpha \frac{\left(y_{k}-s_{k}\right)^{T} F\left(x_{k+1}\right)}{y_{k}^{T} d_{k}} \text { for } k=1,2,3, \cdots, \theta_{k}=\frac{s_{k}^{T} s_{k}}{s_{k}^{T} y_{k}}
$$

The direction $d_{k}$ is assumed to be a decent one and is obtained using

$$
d_{k}=\left\{\begin{array}{c}
-F\left(x_{k}\right) \text { if } k=0 \\
-F\left(x_{k-1}\right)+\beta_{k} d_{k-1} \quad \text { if } k \geq 1
\end{array}\right.
$$

where $\beta_{k}$ is term as conjugate gradient parameter. The CG methods for solving nonlinear systems of equations generates an iterative points $\left\{x_{k}\right\}$ from initial given point $x_{0}$ via

$$
x_{k+1}=x_{k}+\gamma_{k} d_{k}
$$

where $\gamma_{k}>0$ is attained via line search. Using the above procedure, the following is the algorithm for the new update.

\section{Algorithm:}

Step 1: Given $x_{0}, \gamma>0, \alpha=0.6, \sigma \epsilon(0,1)$ and $\epsilon>0$ compute $d_{0}=-F\left(x_{0}\right)$, set $k=0$.

Step 2: Compute $F\left(x_{k}\right)$ and test the stopping criterion, i.e. $\left\|F\left(x_{k}\right)\right\| \leq \epsilon$, if yes, then stop, otherwise continue with next step.

Step 3: Compute $\gamma_{k}$ by using the line search in $[16,17,21,22]$

Step 4: Compute $\theta_{k}=\frac{y_{k}^{T} y_{k}}{y_{k}^{T} s_{k}}$, and new $\beta_{k}$.

Step 5: Compute $x_{k+1}=x_{k}+\alpha_{k} d_{k}$

Step 6: Compute search direction using (4)

Step 7: Set $k=k+1$ and go to step 2 . 


\section{NUMERICAL RESULT}

In this section, the numerical results of the proposed method is given. The proposed hybrid method is denoted as M1, and its performance is compared with the classical conjugate gradient parameter methods denoted as M2 and M3 respectively. In Tables 1 and 2, each n-dimensional problem with initial starting point is considered as one problem, therefore a total of 40 benchmark problems were solved. The computational experiment is based on the number of iterations and CPU time. To ascertain the global convergence of the proposed method, the benchmarks problems in $[21,22]$ was used with two different ISP, and the output of the performance of the methods was based on the performance profile presented by Dolan and More [24].

Table 1. Performance profile of M1, M2 and M3 based on iteration number \& CPU time

\begin{tabular}{ccccccccc}
\hline Prob & ISP & Dim & M1 & & M2 & & M3 \\
& & & Iter & CPU & Iter & CPU & Iter & CPU \\
\hline 1a & $(0.5,0.5, \cdots, 0.5)^{T}$ & 10 & 1 & 0.006806 & 13 & 0.015034 & 17 & 0.014147 \\
& & 100 & 1 & 0.000632 & 14 & 0.002887 & 18 & 0.002249 \\
& & 500 & 1 & 0.000366 & 14 & 0.003004 & 18 & 0.008648 \\
& & 1000 & 1 & 0.000433 & 14 & 0.003024 & 19 & 0.009221 \\
& & 5000 & 1 & 0.001403 & 14 & 0.123704 & 21 & 0.057777 \\
$1 \mathrm{~b}$ & $(0.2,0.2, \cdots, 0.2)^{T}$ & 10 & 5 & 0.000567 & 10 & 0.001078 & 15 & 0.001418 \\
& & 100 & 6 & 0.001142 & 10 & 0.001165 & 18 & 0.003607 \\
& & 500 & 6 & 0.001000 & 11 & 0.001951 & 18 & 0.004433 \\
& & 1000 & 6 & 0.001395 & 11 & 0.004255 & 18 & 0.007740 \\
& & 5000 & 7 & 0.006488 & 11 & 0.013589 & 21 & 0.055354 \\
& & 10 & 6 & 0.000580 & 4 & 0.000809 & 18 & 0.001765 \\
& $(0.5,0.5, \cdots, 0.5)^{T}$ & 100 & 6 & 0.000565 & 4 & 0.000893 & 19 & 0.002758 \\
& & 500 & 6 & 0.000904 & 5 & 0.001237 & 20 & 0.004726 \\
& & 1000 & 6 & 0.001257 & 5 & 0.001637 & 21 & 0.008809 \\
& & 5000 & 6 & 0.006039 & 6 & 0.006157 & 22 & 0.056553 \\
$2 \mathrm{~b}$ & $(0.2,0.2, \cdots, 0.2)^{T}$ & 10 & 4 & 0.000594 & 5 & 0.002852 & 15 & 0.002374 \\
& & 100 & 5 & 0.000736 & 6 & 0.000828 & 15 & 0.002038 \\
& & 500 & 5 & 0.000884 & 6 & 0.001150 & 16 & 0.004103 \\
& & 1000 & 5 & 0.001982 & 6 & 0.001678 & 16 & 0.007631 \\
& & 5000 & 5 & 0.005914 & 7 & 0.005991 & 18 & 0.045659 \\
\hline
\end{tabular}

Table 2. Performance profile of M1, M2 and M3 based on iteration number \& CPU time (cont)

\begin{tabular}{ccccccccc}
\hline Prob & ISP & Dim & M1 & & M2 & & M3 & \\
& & & Iter & CPU & Iter & CPU & Iter & CPU \\
\hline $3 \mathrm{a}$ & $(0.5,0.5, \cdots, 0.5)^{T}$ & 10 & 6 & 0.000583 & 4 & 0.000748 & 18 & 0.001662 \\
& & 100 & 6 & 0.000502 & 4 & 0.000791 & 19 & 0.002683 \\
& & 500 & 6 & 0.000705 & 5 & 0.001285 & 20 & 0.004508 \\
& & 1000 & 6 & 0.000978 & 5 & 0.001404 & 21 & 0.007613 \\
& & 5000 & 6 & 0.004791 & 6 & 0.005932 & 22 & 0.049457 \\
$3 \mathrm{~b}$ & $(0.2,0.2, \cdots, 0.2)^{T}$ & 10 & 4 & 0.000597 & 5 & 0.000625 & 15 & 0.001215 \\
& & 100 & 5 & 0.000629 & 6 & 0.000764 & 15 & 0.001608 \\
& & 500 & 5 & 0.000863 & 6 & 0.001065 & 16 & 0.003806 \\
& & 1000 & 5 & 0.001085 & 6 & 0.002203 & 16 & 0.006194 \\
& & 5000 & 5 & 0.005834 & 7 & 0.005344 & 18 & 0.048274 \\
$4 \mathrm{a}$ & $(0.5,0.5, \cdots, 0.5)^{T}$ & 10 & 3 & 0.000345 & 1 & 0.009970 & 51 & 0.005631 \\
& & 100 & 3 & 0.000391 & 1 & 0.000614 & 63 & 0.008490 \\
& & 500 & 3 & 0.000423 & 1 & 0.000783 & 69 & 0.019573 \\
& & 1000 & 4 & 0.000483 & 1 & 0.001370 & 73 & 0.032623 \\
& & 5000 & 4 & 0.001014 & 1 & 0.004664 & 79 & 0.149735 \\
$4 \mathrm{~b}$ & $(0.2,0.2, \cdots, 0.2)^{T}$ & 10 & 2 & 0.105179 & $\mathrm{~F}$ & 0.000493 & 60 & 0.006403 \\
& & 100 & 4 & 0.126227 & $\mathrm{~F}$ & 0.000784 & 70 & 0.013660 \\
& & 500 & 4 & 0.212208 & $\mathrm{~F}$ & 0.000963 & 78 & 0.022243 \\
& & 1000 & 4 & 0.334854 & $\mathrm{~F}$ & 0.005148 & 80 & 0.033186 \\
& & 5000 & 4 & 1.369668 & $\mathrm{~F}$ & 0.004322 & 88 & 0.159905 \\
\hline
\end{tabular}

The performance profile $P: R \rightarrow[0,1]$ is defined as follows: Let $P$ and $S$ be the set of problems and set of solvers respectively. For $n_{s}$ solvers and $n_{p}$ problems, and for each problem $p \in P$ and for each solver $s \in S, t_{p, s}:=$ (number of iterations required to solve problem $p$ by solver $s$ ) is defined. The performance ratio is given by: 


$$
r_{p, s}:=t_{p, s} / \min \left\{t_{p, s}\right\}
$$

Then the performance profile is defined by:

$$
P(\tau):=\frac{1}{n_{p}} \operatorname{size}\left\{p \in P: r_{p, s} \leq \tau\right\},
$$

for all $\tau \in R$ where $P(\tau)$ is the probability for solver $s \in S$ that a performance ratio $r_{p, s}$ is within a factor $\tau \in R$ of the best possible ratio. The code for the proposed method was done using MATLAB 7.1, R2009b programming environment and run on a personal computer $2.4 \mathrm{GHz}$, Intel (R) Core (TM) i7-5500U CPU processor, 4GB RAM memory and on windows XP operator. Both the methods was implemented with the same parameters as $\gamma_{1}=0.01, r=0.2, \sigma_{1}=\sigma_{2}=10^{-4}$, and $\eta_{k}=\frac{1}{\{k+1\}^{2}}$. The search is stopped if: (i) ॥ $F\left(x_{k}\right) \|<\epsilon$ with $\epsilon<10^{-4}$ or (ii) The total number of iteration exceeds 1000 .

The meaning of each column in the tables are stated as follows, "P": stands for Benchmark problem, "ISP": stands for initial starting points, "n": stands for dimension of the test problems, "Iter": the total number of iterations and "CPU": the processing time in seconds. A particular problem i, performs better if the number of iteration (iter) and/or the CPU time in seconds (Time) is less than the number of iteration or the CPU time corresponding to the other methods respectively.

From the above tables, the proposed method outperformed the two other methods in terms of CPU times and number of iterations, as shown in Figures 1 and 2.

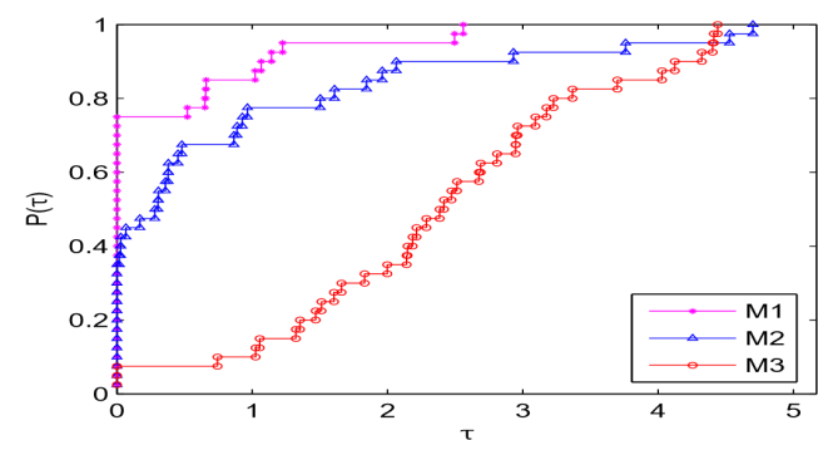

Figure 1. Performance profile of M1, M2 and M3 with respect to the number of iterations

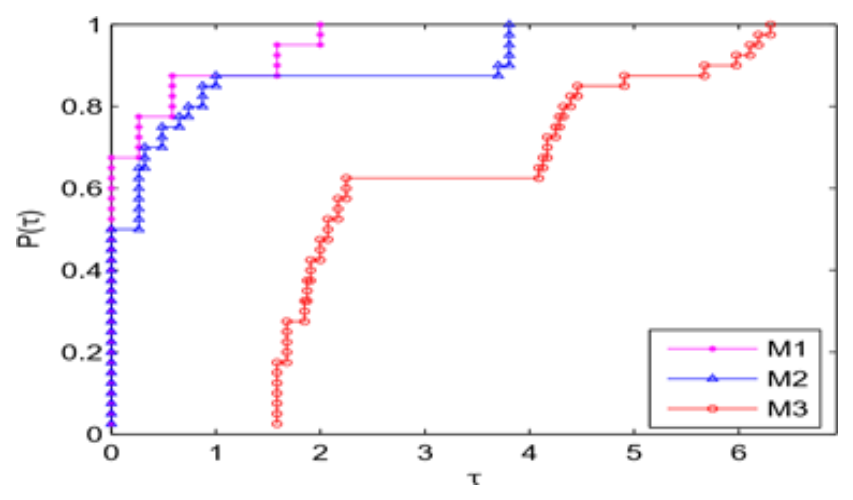

Figure 2. Performance profile of M1, M2 and M3 with respect to the CPU time

\section{CONCLUSION AND FUTURE WORK}

In this article, a derivative free method for solving symmetric nonlinear systems of equations is given. The hybrid method achieved its objectives fully, i.e. it is faster in terms of processing time and effective in terms of number of iterations. Thus, the hybrid conjugate gradient parameter is a very good alternative for solving symmetric nonlinear systems of equations. For further research work, the method can be modified to solve nonsmooth nonlinear equations. 


\section{ACKNOWLEDGEMENTS}

The authors would like to thank the Government of Malaysia for funding this research under the Fundamental Research Grant Scheme (FRGS/1/2018/ICT03/UNISZA/02/2) and also the Research Management, Innovation and Commercialization of Universiti Sultan Zainal Abidin.

\section{REFERENCES}

[1] J.C. Gilbert and J. Nocedal, Global convergence properties of conjugate gradient methods for optimization, SIAM J. Optim. 2 (1992), pp. 21-42.

[2] W.W. Hager and H. Zhang, A survey of nonlinear conjugate gradient methods, Pac. J. Optim. 2 (2006), pp. 35-58.

[3] G. Li, C. Tang, and Z. Wei, New conjugacy condition and related new conjugate gradient methods for unconstrained optimization, J. Comput. Appl. Math. 202 (2007), pp. 523-539.

[4] W. Zhou and D. Shen, "An inexact PRP conjugate gradient method for symmetric nonlinear equations," Numerical Functional Analysis and Optimization, vol. 35, no. 3, pp. 370-388, 2014.

[5] M.J.D. Powell, Nonconvex Minimization calculations and the conjugate gradient method, in Numerical Analysis (Dundee, 1983), Lecture Notes in Mathematics, Vol. 1066, D.F. Griffiths, ed., Springer, Berlin, 1984, pp. 122-141.

[6] D. Touati-Ahmed and C. Storey, Efficient hybrid conjugate gradient techniques, J. Optim. Theory Appl. 64 (1990), pp. 379-397.

[7] Y.F. Hu and C. Storey, Global convergence result for conjugate gradient methods, J. Optim. Theory Appl. 71 (1991), pp. 399-405.

[8] Y.H. Dai and Y. Yuan, An efficient hybrid conjugate gradient method for unconstrained optimization, Ann. Oper. Res. 103 (2001), pp. 33-47.

[9] N. Andrei, A hybrid conjugate gradient algorithm for unconstrained optimization as a convex combination of Hestenes-Stiefel and Dai-Yuan, Stud. Inform. Control 17 (2008), pp. 55-70.

[10] N. Andrei, Accelerated hybrid conjugate gradient algorithm with modified secant condition for unconstrained optimization, Numer. Algor. 54 (2010), pp. 23-46.

[11] Ibrahim Abdullahi and Rohanin Ahmad, (2017). Global Convergence Analysis of a new Hybrid Conjugate Gradient Method for Unconstrained Optimization Problems, Malaysian Journal of Fundamental and Applied Sciences, 13(2) 40-48.

[12] Wan Farah Hanan Wan Osman, Mohd Asrul Hery Ibrahim and Mustafa Mamat, (2017). Hybrid DFP-CG Method for Solving Unconstrained Optimization Problems, Journal of Physics, Conference Series, doi:10.1088/17426596/890/1/012033

[13] Shengwei Yao and Bin Qin (2014). A Hybrid of DL and WYL Nonlinear Conjugate Gradient Methods, Abstract and Applied Analysis, Volume, Article ID 279891, 9 pages, http://dx.doi.org/10.1155/2014/279891.

[14] J. Nocedal and S.J. Wright, Numerical Optimization, Springer, New York, 2006.

[15] W. Sun and Y.X. Yuan, Optimization Theory and Methods: Nonlinear Programming, Springer, New York, 2006.

[16] M. Y. Waziri and S. Jamilu, (2015). A Derivative-Free Conjugate Gradient Method and Its Global Convergence for Solving Symmetric Nonlinear Equations, International Journal of Mathematics and Mathematical Sciences, 39: Article ID 961487, 10 pages.

[17] M. K. Dauda, Mustafa Mamat, Mohamad Afendee Mohamed, Fatma Susilawati Mohamad and M.Y. Waziri, (2017). Derived Conjugate Gradient Parameter for Solving Symmetric Systems of Nonlinear Equations, Far East Journal of Mathematical Sciences (FJMS), 102 (11)2599-2610.

[18] S. Babaie-Kafaki, A modified BFGS algorithm based on a hybrid secant equation, Sci. China Math. 54 (2011), pp. 2019-2036.

[19] S. Babaie-Kafaki, M. Fatemi, and N. Mahdavi-Amiri, Two effective hybrid conjugate gradient algorithms based on modified BFGS updates, Numer. Algor. 58 (2011), pp. 315-331.

[20] S. B. Kafaki, A hybrid conjugate gradient method based on a quadratic relaxation of the Dai-Yuan hybrid conjugate gradient parameter, A Journal of Mathematical Programming and Operations Research, Vol. 62, No.7, 929-941, (2011).

[21] M. K. Dauda, Mustafa Mamat, M. Y. Waziri, Fadhila Ahmad and Fatma Susilawati Mohamad, (2016). Inexact CGMethod via SR1 Update for Solving Systems of Nonlinear Equations, Far East Journal of Mathematical Sciences (FJMS) 100, (11) 1787-1804.

[22] D. H. Li and M. Fukushima, A derivative-free line search and global convergence of Broyden-like methods for nonlinear equations, Optimization Methods and Software, 13 (2000), 181-201.

[23] M.K. Dauda, Mustafa Mamat, M.Y. Waziri, Fadhilah Ahmad and Fatma Susilawati Mohamad, (2016), Improved Quasi-Newton Method Via PSB Update for Solving Systems of Nonlinear equations, AIP Conference Proceedings (ICOQSIA 2016), 1782, 030009 (2016); doi: 10.1063/1.4966066.

[24] E. Dolan and J. Mor'e, Benchmarking optimization software with performance profiles, Math. Program. Ser. A, 91 (2002), 201-213.

[25] Y.H. Dai and Y. Yuan, An efficient hybrid conjugate gradient method for unconstrained optimization, Ann. Oper. Res. 103 (2001), pp. 33-47. 